\title{
Acute Risk Change for Cardiothoracic Admissions to Intensive Care (ARCTIC index): A new measure of quality in cardiac surgery
}

\author{
Tim G. Coulson, BM, BSc, ${ }^{\mathrm{a}, \mathrm{c}}$ Michael Bailey, PhD, MSc, ${ }^{\mathrm{b}}$ Christopher M. Reid, PhD, \\ Lavinia Tran, PhD, BBiomedsci, ${ }^{c}$ Daniel V. Mullany, MBBS, MMedSc, FCICM, FANZCA, \\ Julian A. Smith, MS, FRACS, ${ }^{\mathrm{e}}$ and David Pilcher, MBBS, MRCP, FRACP, FCICM ${ }^{\mathrm{a}, \mathrm{f}, \mathrm{f}}$
}

\begin{abstract}
Background: Quality of cardiac surgical care may vary between institutions. Mortality is low and large numbers are required to discriminate between hospitals. Measures other than mortality may provide better comparisons.

Objectives: To develop and assess the Acute Risk Change for Cardiothoracic Admissions to Intensive Care (ARCTIC) index, a new performance measure for cardiothoracic admissions to intensive care units (ICUs).

Methods: The Australian and New Zealand Society of Cardiac and Thoracic Surgeons database and Australian and New Zealand Intensive Care Society Adult Patient Database were linked. Logistic regression was used to generate a predicted risk of death first from preoperative data using the previously validated Allprocscore and second on admission to an ICU using Acute Physiology and Chronic Health Evaluation III score. Change in risk as a percentage (ARCTIC) was calculated for each patient. The validity of ARCTIC as a marker of quality was assessed by comparison with intraoperative variables and postoperative morbidity markers.
\end{abstract}

Results: Sixteen thousand six hundred eighty-seven patients at 21 hospitals from 2008 to 2011 were matched. An increase in ARCTIC score was associated with prolonged cardiopulmonary bypass time $(P=.001)$, intraoperative blood product transfusion $(P<.001)$, reoperation $(P<.0001)$, postoperative renal failure $(P<.0001)$, prolonged ventilation $(P<.0001)$, and stroke $(P=.001)$.

Conclusions: The ARCTIC index is associated with known markers of perioperative performance and postoperative morbidity. It may be used as an overall marker of quality for cardiac surgery. Further work is required to assess ARCTIC as a method to discriminate between cardiac surgical units. (J Thorac Cardiovasc Surg 2014;148:3076-81)

See related commentary on pages 3082-3.

Supplemental material is available online.

Cardiac surgery programs in many countries routinely monitor procedures and outcomes. The function of this large-scale monitoring is to maintain and improve the quality of patient care. Despite more than 20 years of

From the Department of Intensive Care, ${ }^{a}$ The Alfred Hospital, Melbourne, Victoria, Australia; Australian and New Zealand Intensive Care Research Centre, Department of Epidemiology and Preventive Medicine, ${ }^{\mathrm{c}}$ School of Public Health and Preventive Medicine, Monash University, Melbourne, Australia; Critical Care Research Group, ${ }^{\mathrm{d}}$ University of Queensland, Brisbane, Australia; Department of Surgery, ${ }^{e}$ Monash University, and Department of Cardiothoracic Surgery, Monash Health, Melbourne, Australia; and ANZICS Centre for Outcome and Resource Evaluation, ${ }^{\mathrm{f}}$ Melbourne, Australia.

Tim Coulson was supported by an Alfred-Monash research fellowship.

Disclosures: Authors have nothing to disclose with regard to commercial support.

Received for publication Jan 28, 2014; revisions received June 2, 2014; accepted for publication June 5, 2014; available ahead of print Aug 16, 2014.

Address for reprints: Tim G. Coulson, BM, BSc, Department of Intensive Care,

The Alfred Hospital, Commercial Rd, VIC 3181, Melbourne, Australia (E-mail: timcoulson@doctors.org.uk).

$0022-5223 / \$ 36.00$

Copyright $($ C 2014 by The American Association for Thoracic Surgery

http://dx.doi.org/10.1016/j.jtcvs.2014.06.069 experience, the concept of quality of care remains difficult to define and measure. ${ }^{1}$ Measures of quality include outcome measures such as risk-adjusted mortality and risk-adjusted major morbidity (eg, renal failure, stroke, deep sternal wound infection, prolonged ventilation, and reoperation) and process measures such as use of internal mammary artery grafts and optimal perioperative medical therapy. Currently the Society of Thoracic Surgeons uses all of the above in a composite quality score. ${ }^{2}$

The most intuitive measures of quality are outcomes. Mortality rate (adjusted for preoperative risk) was 1 of the earliest quality measures to be used. Due to advances in surgical and medical knowledge the in-hospital mortality rate for the most common cardiac surgical procedure, coronary artery bypass graft $(\mathrm{CABG})$, has been reduced to as little as $1.5 \% .^{3}$ This means that using in-hospital mortality to determine significant variation in performance between units requires large numbers. ${ }^{4}$ Achieving these numbers in many units takes considerable time, delaying the time to recognition of under- or overperforming units. Alternative methods may be useful to more effectively monitor and recognize outliers in performance.

The Australian and New Zealand Society of Cardiac and Thoracic Surgeons Cardiac Surgical Database (ANZSCTSCSD) was developed in 2001 and includes data from public 


$\begin{aligned} & \text { Abbreviations and Acronyms } \\ & \text { ANZSCTS-CSD = } \text { Australian and New Zealand } \\ & \text { Society of Cardiac and Thoracic } \\ & \text { Surgeons Cardiac Surgery } \\ & \text { Database } \\ & \text { ANZICS-APD }= \text { Australian and New Zealand } \\ & \text { Intensive Care Society Adult } \\ & \text { Patient Database } \\ &= \text { Acute Physiological and } \\ & \text { Chronic Health Evaluation } \\ &= \text { Acute Risk Change for } \\ & \text { Cardiothoracic Admissions to } \\ & \text { Intensive Care } \\ &= \text { coronary artery bypass graft } \\ &= \text { intensive care unit } \\ &= \text { preoperative predicted risk of } \\ & \text { death } \\ &= \text { postoperative predicted risk of } \\ & \text { death } \\ & \text { CABG } \text { valve repair/replacement }\end{aligned}$

and private hospitals. Patient data from all cardiac surgical procedures occurring at participating hospitals is included. Demographic data; pre-, intra-, and postoperative data; outcomes; and derived scores such as the previously validated Allprocscore $^{5,6}$ are recorded. Cardiac surgical risk of death scores typically use preoperative variables that are not under the control of the provider.

The Australian and New Zealand Intensive Care Society Adult Patient Database (ANZICS-APD) is 1 of 4 registries run by the ANZICS Centre for Outcome and Resource Evaluation. The ANZICS-APD contains de-identified patient data on 1.4 million intensive care unit (ICU) admissions from $85 \%$ of ICUs in Australia and New Zealand. It contains demographic, diagnostic, and physiologic data from the first 24 hours of ICU admission for calculation of severity of illness scores such as the Acute Physiological and Chronic Health Evaluation (APACHE) III score. It can therefore provide information on the immediate postoperative period. Both databases are audited to assess reliability of submitted data. ${ }^{5,7,8}$ The presence of 2 databases with data from the same patients provides a rare opportunity to study the entire perioperative episode, giving a more complete picture of procedure-related risk for each patient.

The aim of our study was to derive a measure of change in mortality risk from the preoperative period to the time of ICU admission after CABG or cardiac valve repair/replacement (VR) surgery (Figure 1) and to attempt to validate this Acute Risk Change for Cardiothoracic Admissions to Intensive Care (ARCTIC) index as a potential marker of perioperative cardiothoracic performance. Our hypothesis was that ARCTIC would not only correlate with intraoperative measures of surgical performance but also postoperative markers of morbidity already recognized as performance measures for cardiothoracic units.

\section{METHODS}

\section{Matching of ANZICS and ANZSCTS Databases}

Probabilistic methodology was used to match individual patient data from 21 sites that contributed to both ANZSCTS databases and the ANZICS-APD between 2008 and 2011. ${ }^{9}$ Only CABG and/or cardiac valve operations were included. Patients in each database were considered matched if a minimum of 6 out of 7 of the following variables were identical: ICU admission date, hospital admission date, ICU discharge date, hospital discharge date, age, sex, and postcode. Patients admitted to an ICU before cardiac surgery who then proceed to cardiac surgery would have a nonoperative APACHE diagnosis were excluded as these patients would not have postoperative APACHE scores.

\section{Generation of ARCTIC}

All statistical analyses were performed using STATA version 12 (StataCorp, College Station, Tex). Preoperative predicted risk of death $\left(\mathrm{ROD}_{\mathrm{pre}}\right)$ was calculated using logistic regression and Allprocscore. Variables in Allprocscore include age, gender, New York Heart Association functional class, urgency of procedure, ejection fraction, hypercholesterolemia, preoperative dialysis, previous cardiac surgery, procedure type, inotropic medication, peripheral vascular disease, and body mass index. Postoperative predicted risk of death $\left(\mathrm{ROD}_{\text {post }}\right)$ was calculated using logistic regression based on APACHE III score and type of operation (CABG, VR, or CABG and VR). The APACHE III score is based on age, chronic health conditions, and acute physiologic variables (eg, blood pressure). Model calibration was checked using the Hosmer-Lemeshow goodness-of-fit test. Model discrimination was assessed using area under the receiver operator characteristic. The ARCTIC index was created by subtraction of $\mathrm{ROD}_{\text {pre }}$ from $\mathrm{ROD}_{\text {post }}$, such that a positive ARCTIC (as a percentage increase in risk of death) denoted an increase in risk of death. Normality of distribution for ARCTIC was assessed.

\section{Relationship of ARCTIC to Pre- and Intraoperative Variables}

The relationship between the ARCTIC index to pre- and intraoperative factors was assessed in the following way: pre- and intraoperative variables (excluding those already used in Allprocscore) were identified. Univariate linear regression was carried out to look for association between variables and ARCTIC. Variables with a univariate $P<.10$ were subjected to stepwise regression (forward and backward elimination procedures) to identify factors independently associated with ARCTIC. Two-way interactions between variables were tested. Variables exhibiting colinearity were removed.

\section{Relationship of the ARCTIC Index to Postoperative Morbidity}

The ARCTIC index was compared with other known markers of morbidity, ${ }^{2}$ including new renal failure (increased creatinine $>200 \mu \mathrm{mol} / \mathrm{L}$, doubling of baseline creatinine, or new need for renal replacement therapy), prolonged ventilation ( $>24$ hours), return to operating theater (for complications related to original surgery), deep sternal wound infection (debridement plus antibiotics or positive culture), and new stroke (deficit $>72$ hours). The mean ARCTIC 


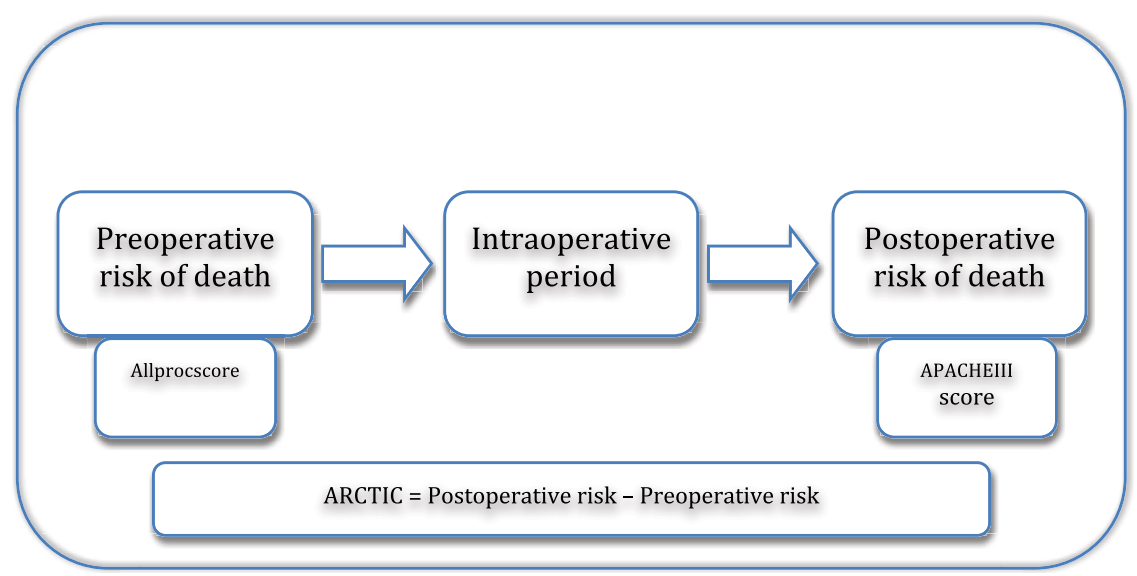

FIGURE 1. The Acute Risk Change for Cardiothoracic Admissions to Intensive Care (ARCTIC) index. Preoperative risk was calculated using Allprocscore, whereas postoperative risk was calculated using Acute Physiology and Chronic Health Evaluation (APACHE III) score.

for groups with and without the complication was compared using $t$ tests.

\section{Ethical Review}

The study was reviewed and approved by the Alfred Hospital research ethics committee (Ref 66/13).

\section{RESULTS}

Twenty-seven thousand one hundred fifteen patients were identified in the ANZSCTS-CSD with a valid ICU admission date. Nineteen thousand three hundred four patients could be matched using at least 6 identical

ANZICS-APD

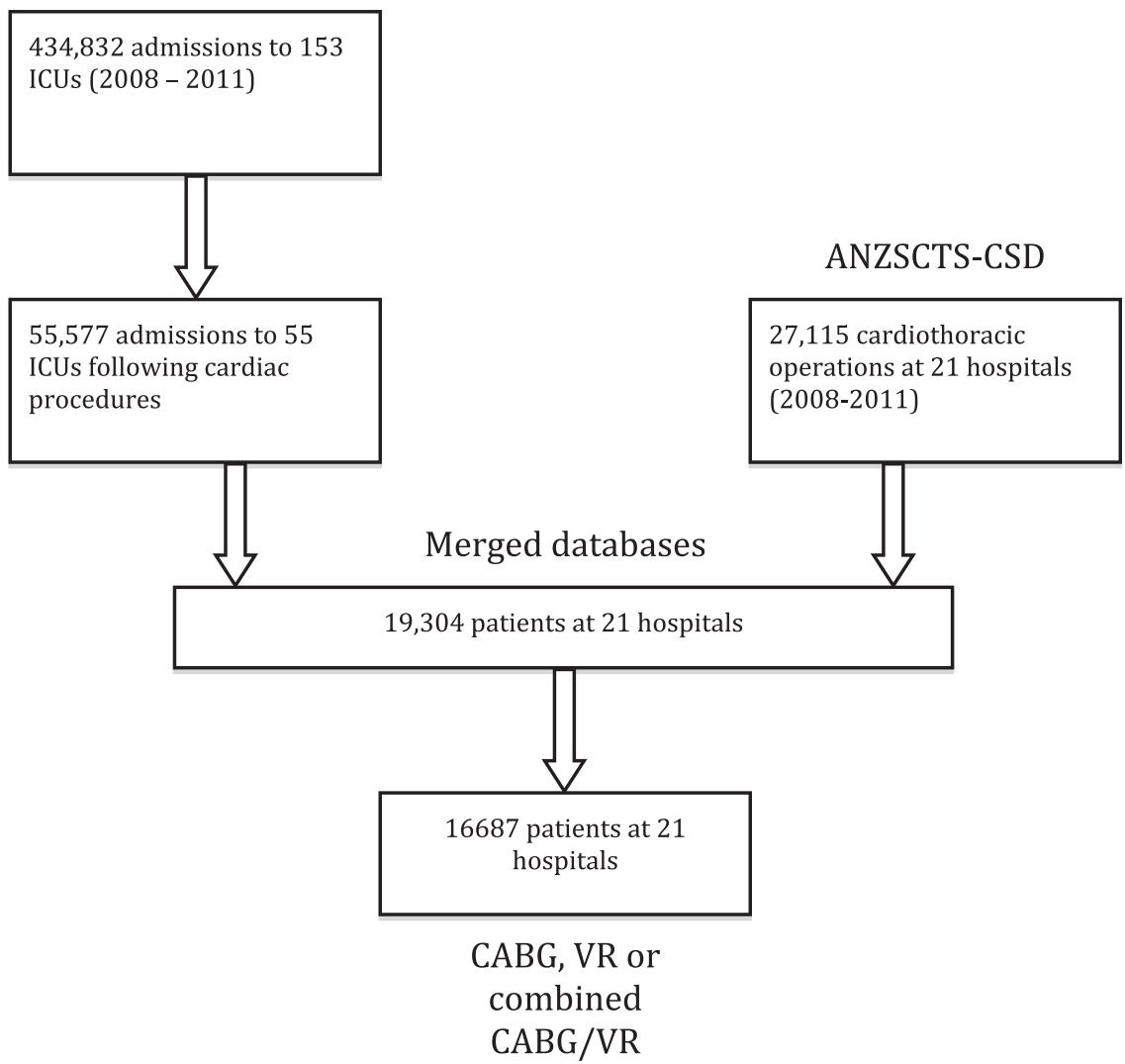

FIGURE 2. Consort flow diagram showing original databases and final merged database. ANZICS-APD, Australian and New Zealand Intensive Care Society Adult Patient Database; ICUs, intensive care units; ANZSCTS-CSD, Australian and New Zealand Society of Cardiac and Thoracic Surgeons Cardiac Surgery Database; $C A B G$, coronary artery bypass graft; $V R$, valve repair/replacement. 
TABLE 1. Patient demographics, risk scores, and perioperative variables stratified according to survival status

\begin{tabular}{|c|c|c|c|c|}
\hline Characteristic & Survived $(\mathrm{n}=16,326)$ & Died $(\mathbf{n}=325)$ & All patients $(\mathrm{n}=16,687)^{*}$ & $P$ value \\
\hline Age, $y$ & $65.8 \pm 12.5$ & $72.0 \pm 11.3$ & $65.9 \pm 12.5$ & $<.0001$ \\
\hline Length of stay & $9(7-15)$ & $15(6-32)$ & $9(7-15)$ & $<.0001$ \\
\hline Male & 73.2 & 60.1 & 72.9 & $<.0001$ \\
\hline Valve repair & 25.6 & 29.3 & 25.7 & .18 \\
\hline Coronary artery bypass graft & 62.6 & 45.2 & 62.2 & $<.0001$ \\
\hline Ventricular repair/coronary artery bypass graft & 11.8 & 25.5 & 12.1 & $<.0001$ \\
\hline Allprocscore & $5.21 \pm 4.7$ & $11.20 \pm 6.1$ & $5.34 \pm 4.85$ & $<.0001$ \\
\hline Preoperative predicted risk of death & 1.85 & 6.94 & 1.95 & $<.0001$ \\
\hline Acute Physiology and Chronic Health Evaluation III score & $46 \pm 16$ & $75 \pm 29$ & $46 \pm 17$ & $<.0001$ \\
\hline Postoperative predicted risk of death & 1.69 & 14.1 & 1.94 & $<.0001$ \\
\hline $\begin{array}{l}\text { Acute Risk Change for Cardiothoracic Admissions to } \\
\text { Intensive Care index score }\end{array}$ & -0.16 & 7.27 & -0.01 & $<.0001$ \\
\hline Red cell units transfused & $0(0-2)$ & $6(2-12)$ & $0(0-2)$ & $<.0001$ \\
\hline Plasma units transfused & $0(0-0)$ & $3(0-8)$ & $0(0-0)$ & $<.0001$ \\
\hline Cardiopulmonary bypass time, min & $101(77-132)$ & $143(98-201)$ & $101(77-134)$ & $<.0001$ \\
\hline Diabetes & 30.3 & 38.1 & 30.4 & .003 \\
\hline Preoperative creatinine & $85(72-102)$ & $98(80-129)$ & $85(72-102)$ & $<.0001$ \\
\hline Ventricular assist device used & 0.06 & 5.64 & 0.16 & $<.001$ \\
\hline Balloon pump used & 4.0 & 31.8 & 4.6 & $<.001$ \\
\hline Antifibrinolytics used & 71.4 & 80.8 & 71.6 & $<.001$ \\
\hline Consultant operating & 83.8 & 88.4 & 83.9 & .05 \\
\hline Cardiogenic shock & 1.03 & 7.81 & 1.17 & $<.001$ \\
\hline New renal failure & 4.1 & 38.6 & 4.8 & $<.001$ \\
\hline Prolonged ventilation & 9.6 & 58.9 & 10.6 & $<.001$ \\
\hline New stroke & 0.8 & 13.2 & 1.1 & $<.001$ \\
\hline Return to operating theater & 5.8 & 41.4 & 6.5 & $<.001$ \\
\hline Deep sternal wound infection & 0.7 & 4.1 & 0.8 & $<.001$ \\
\hline
\end{tabular}

Values are given as percent, mean \pm standard deviation, or median (interquartile range). Percentages relate to column population. $*$ Survival data was missing for 36 patients.

variables. There were differences between matched and unmatched patients that achieved statistical significance (Appendix Table E1). After removal of non-CABG/VR procedures 16,687 patients at 21 centers were left in the database (Figure 2). Demographics and overall outcomes of study patients are shown in Table 1 .

Logistic regression models generated using Allprocscore and APACHE III score performed well in predicting $\mathrm{ROD}_{\text {pre }}$ (area under receiver operating curve, 0.78; Hosmer-Lemeshow $\chi^{2}, 12.56 ; P=.13$ ) and $\mathrm{ROD}_{\text {post }}$ (area under receiver operating curve, 0.84; Hosmer-Lemeshow $\left.\chi^{2}, 10.4 ; P=.24\right)$. ARCTIC $\left(\mathrm{ROD}_{\mathrm{post}}-\mathrm{ROD}_{\mathrm{pre}}\right)$ assumed a symmetrical distribution.

Twenty three pre- and intraoperative variables were identified as having a significant univariate relationship with ARCTIC, including race, diabetes, preoperative creatinine, hypertension, hypercholesterolemia, preoperative shock, immunosuppressive treatment, infective endocarditis, clopidogrel use, tirofiban use, cardiopulmonary bypass time, crossclamp time, presence of consultant surgeon, red cell units transfused, fresh frozen plasma units transfused, platelets transfused, antifibrinolytic agent use, intraoperative transoesophageal echocardiography, intraoperative decision to graft coronary arteries, number of diseased vessels, intra-aortic balloon pump use, and ventricular assist device use. Stepwise regression resulted in the identification of 10 variables independently associated with ARCTIC. All variables were available for $87 \%$ of the matched database. Bypass time and crossclamp time exhibited a high degree of collinearity and hence crossclamp time was eliminated from the model. The interaction between shock and red blood cells transfused was found to be significant. The final model is shown in Table 2. Seven variables were independently associated with risk increase. Four, including the 1 interaction variable, were associated with a reduction in risk.

ARCTIC was significantly higher with 4 of 5 of the postoperative morbidity markers tested: new renal failure $(-0.12 \%$ [standard deviation, 0.03 ] vs $2.23 \%$ [standard deviation, 0.43$] ; \quad P<.0001$ ), prolonged ventilation ( $-0.19 \%$ [standard deviation, 0.03 ] vs $1.47 \%$ [standard deviation, 0.27]; $P<.0001$ ), return to operating theater ( $-0.17 \%$ [standard deviation, 0.04 ] vs $2.28 \%$ [standard deviation, 0.35 ]; $P \leq .0001)$ and new stroke $(-0.03 \%$ [standard deviation, 0.04 ] vs $1.19 \%$ [standard deviation, 0.70 ]; $P=.001$ ) (Table 3). Deep sternal wound infection was not significantly associated with risk change.

\section{DISCUSSION}

We have developed the ARCTIC index, which represents a change in risk of death from the preoperative to 
TABLE 2. Regression model: Variables with positive coefficients are associated with an increase in predicted risk of death (positive ARCTIC); those with negative coefficients are associated with a reduction

\begin{tabular}{lccr}
\hline \multicolumn{1}{c}{ Risk change } & Coefficient & Standard error & $\boldsymbol{P}$ value \\
\hline Red cells transfused $^{*}$ & 0.19 & 0.02 & $<.001$ \\
Fresh frozen plasma transfused $^{*}$ & 0.049 & 0.02 & .032 \\
Bypass time & 0.18 & 0.05 & .001 \\
Diabetic patient & 0.31 & 0.09 & .001 \\
Preoperative creatinine & 0.0039 & 0.0005 & $<.001$ \\
Ventricular assist device used* & 15.94 & 0.96 & $<.001$ \\
Intra-aortic balloon pump used* & 0.80 & 0.21 & $<.001$ \\
Antifibrinolytics used & -0.43 & 0.10 & $<.001$ \\
Consultant surgeon operating & -0.52 & 0.11 & $<.001$ \\
Cardiogenic shock* & -2.95 & 0.49 & $<.001$ \\
Red blood cells/cardiogenic & -0.39 & 0.05 & $<.001$ \\
\multicolumn{1}{c}{ shock interaction } & & & \\
\hline N=14,496. ARCTIC, Acute Risk Change for Cardiothoracic Admissions to Intensive \\
Care. *At any perioperative point. & & &
\end{tabular}

postoperative period for patients undergoing cardiac surgery. We have shown consistent and biologically plausible relationships with known perioperative markers of morbidity. To our knowledge this is the first time a potential overall measure of cardiac performance has been developed that is relevant to the whole perioperative period.

By combining cardiac and ICU databases we were able to calculate both a pre- and postoperative risk for patients. Both risk scores performed well and enabled the calculation of change in mortality risk (the ARCTIC index) between the pre- and postoperative periods. Although it was not surprising that the previously published Allprocscore ${ }^{5}$ was related to mortality, the APACHE III score has not until now been validated for this purpose. Indeed the APACHE III score had better discrimination than the Allprocscore, which may relate to the fact it is measured later in a patient's course (ie, temporally closer to the final outcome). Intraoperative events and preoperative risk factors that are known to be associated with poor outcome were associated with an adverse ARCTIC index score (ie, an increasing postoperative risk of death). These included the number of red cell and plasma units transfused, cardiac bypass time, intra-aortic balloon pump use, preoperative creatinine level, and diabetes. ${ }^{10-16}$ It is also unsurprising that the requirement for a ventricular assist device insertion would be associated with an adverse ARCTIC index score. Thus the relationship between ARCTIC and these intraoperative events supports consideration for its use as a plausible marker for assessing perioperative performance.

Similarly some intraoperative variables were associated with a beneficial ARCTIC index score, or reduction in postoperative risk (ie, those with negative coefficients); for example, a consultant surgeon operating (as opposed to a more junior member of the team) and the use of any antifibrinolytic agent (previously shown to be associated with reduction in bleeding ${ }^{17}$ ). Cardiogenic shock was also associated with a reduction in postoperative risk. This may be due to therapeutic intervention in patients with preoperative cardiogenic shock and a very high preoperative risk of death, or the known overprediction of cardiac surgical risk models in very high risk patients ${ }^{18}$ or unquantifiable aspects of patient selection. It should also be noted that the database records cardiogenic shock at any perioperative point and therefore this variable might include a heterogenous population. A statistical interaction between cardiogenic shock and red cell units transfused was also associated with ARCTIC. The nature of this interaction appears to be a positive effect of red cells transfused when shock is present and a negative effect when shock is absent. Although the benefits and adverse effects of transfusion are only partially understood, they are likely to have differential effects according to clinical situation; therefore, this finding does seem at least physiologically reasonable. One other study has reported a trend toward increased cardiogenic shock in patients with restrictive transfusion regimens. ${ }^{19}$

Patients with higher ARCTIC index score were more likely to experience postoperative markers of morbidity, including return to the operating theater, prolonged ventilation, stroke, and new renal failure. This further supports the assertion that ARCTIC is associated with an adverse perioperative course leading to postoperative complications. The causes of these adverse events can only be speculated, but given the factors associated with ARCTIC these may include prolonged surgery, less skilled operators, and perioperative bleeding.

Future uses of ARCTIC include as a screening tool to identify near-miss scenarios and to potentially quantify the effect of a complication in a more objective way. For example, take-back to an operating room may not have an adverse effect on risk of death if it is timely. Similarly a prolonged surgical procedure may not be detrimental if a

TABLE 3. ARCTIC percentage by presence or absence of complications

\begin{tabular}{lccc}
\hline \multicolumn{1}{c}{ Complication } & Risk change if complication absent & Risk change if complication present & $\boldsymbol{P}$ value \\
\hline New renal failure & $-0.12 \% \pm 0.03 \%$ & $2.23 \% \pm 0.43 \%$ & $<.0001$ \\
Prolonged ventilation & $-0.19 \% \pm 0.03 \%$ & $1.47 \% \pm 0.27 \%$ & $<.0001$ \\
Return to operating theater & $-0.17 \% \pm 0.04 \%$ & $2.28 \% \pm 0.35 \%$ & $<.0001$ \\
New stroke & $-0.03 \% \pm 0.04 \%$ & $1.19 \% \pm 0.70 \%$ & .001 \\
Deep sternal wound infection & $-0.017 \% \pm 0.04 \%$ & $0.74 \% \pm 0.51 \%$ & .053 \\
\hline
\end{tabular}

Values are given as mean \pm standard deviation. ARCTIC, Acute Risk Change for Cardiothoracic Admissions to Intensive Care. 
patient arrives in the ICU in good physiologic condition and postoperative risk of death is unchanged compared with their preoperative risk. ARCTIC may also be useful in assessing overall comparative performance of cardiothoracic units. Its consistent relationship with many recognized markers of perioperative morbidity may allow its use to benchmark units with very low mortality rates or with very low case numbers. However, further work will be required to determine its applicability to these situations.

\section{Study Limitations}

Limitations should be addressed. Not all patients in the database were matched. Appendix Table E1 shows that there were some small differences between identically matched and unmatched patients that were statistically significant. Matched patients were younger, had lower preoperative risk scores, had lower ejection fractions, and were more likely to be undergoing dialysis. It is unclear why this pattern of matching should occur based on the matching criteria described above. These differences are likely to affect the generalizability of the findings to the overall population. Future data collection should use unique identifiers to reduce the influence of this limitation.

APACHE III score is currently not collected by ANZSCTS so additional data or a regular match would be required to use ARCTIC as a quality marker. This is feasible in Australia and New Zealand; however, the applicability of this score internationally is unknown. Similar data are collected; for example, the European System for Cardiac Operative Risk Evaluation, and future work may examine the applicability of the ARCTIC index to these scores. The time of occurrence of some variables was not recorded; for example, cardiogenic shock and transfusion; therefore, intraoperative events are harder to isolate. The APACHE III score is calculated from the worst variables in the first 24 hours; therefore, change in risk may represent events and treatments within the ICU.

\section{CONCLUSIONS}

The ARCTIC index is a new and potentially useful method to measure quality in cardiac surgery. Unlike most other measures it focuses specifically on perioperative care. It is associated with known markers of intraoperative performance and postoperative morbidity. We have demonstrated the feasibility of development of novel performance measures by matching methods across 2 large datasets. Further work is planned to assess the ARCTIC index as a method to discriminate between cardiac surgical units.

\section{References}

1. Birkmeyer JD, Dimick JB, Birkmeyer NJO. Measuring the quality of surgical care: structure, process, or outcomes? No competing interests declared. J Am Coll Surg. 2004;198:626-32.

2. Shahian DM, Grover FL, Anderson RP, Edwards FH. Quality measurement in adult cardiac surgery: introduction. Ann Thorac Surg. 2007;83:S1-2.

3. Reid CM, Brennan AL, Dinh DT, Billah B, Costolloe CB, Shardey GC, et al. Measuring safety and quality to improve clinical outcomes-curren activities and future directions for the Australian Cardiac Procedures Registry Med J Aust. 2010;193(8 Suppl):S107-10.

4. Dimick JB, Welch HG, Birkmeyer JD. Surgical mortality as an indicator of hospital quality: the problem with small sample size. JAMA. 2004;292:847-51.

5. Billah B, Reid CM, Shardey GC, Smith JA. A preoperative risk prediction model for 30-day mortality following cardiac surgery in an Australian cohort. Eur J Cardiothorac Surg. 2010;37:1086-92.

6. Reid C, Billah B, Dinh D, Smith J, Skillington P, Yii M, et al. An Australian risk prediction model for 30-day mortality after isolated coronary artery bypass: the AusSCORE. J Thorac Cardiovasc Surg. 2009;138:904-10.

7. Centre for Outcome and Resource Evaluation annual report 2011-2012. Melbourne: Australian and New Zealand Intensive Care Society; 2013:1-40.

8. Stow PJ, Hart GK, Higlett T, George C, Herkes R, McWilliam D, et al. Development and implementation of a high-quality clinical database: the Australian and New Zealand Intensive Care Society Adult Patient Database. J Crit Care. 2006;21:133-41.

9. Bohensky MA, Jolley D, Sundararajan V, Pilcher DV, Evans S, Brand CA. Empirical aspects of linking intensive care registry data to hospital discharge data without the use of direct patient identifiers. Anaesth Intensive Care. 2011; 39:202-8.

10. Murphy GJ, Reeves BC, Rogers CA, Rizvi SIA, Culliford L, Angelini GD. Increased mortality, postoperative morbidity, and cost after red blood cell transfusion in patients having cardiac surgery. Circulation. 2007;116:2544-52.

11. Bilgin YM, van de Watering LMG, Versteegh MIM, van Oers MHJ Vamvakas EC, Brand A. Postoperative complications associated with transfusion of platelets and plasma in cardiac surgery. Transfusion. 2011;51:2603-10.

12. Nissinen J, Biancari F, Wistbacka JO, Peltola T, Loponen P, Tarkiainen P, et al Safe time limits of aortic cross-clamping and cardiopulmonary bypass in adult cardiac surgery. Perfusion. 2010;24:297-305.

13. Mukherjee D. Perioperative Cardiac assessment for noncardiac surgery: eight steps to the best possible outcome. Circulation. 2003;107:2771-4.

14. Michalopoulos A, Tzelepis G, Dafni U, Geroulanos S. Determinants of hospital mortality after coronary artery bypass grafting. Chest. 1999;115:1598-603.

15. Sniecinski RM, Levy JH. Bleeding and management of coagulopathy. J Thorac Cardiovasc Surg. 2011;142:662-7.

16. Ranucci M, Castelvecchio S, Conte M, Megliola G, Speziale G, Fiore F, et al. The easier, the better: age, creatinine, ejection fraction score for operative mortality risk stratification in a series of 29,659 patients undergoing elective cardiac surgery. J Thorac Cardiovasc Surg. 2011;142:581-6.

17. Dhir A. Antifibrinolytics in cardiac surgery. Ann Card Anaesth. 2013;16:117.

18. Bhatti F, Grayson AD, Grotte G, Fabri BM, Au J, Jones M, et al. The logistic EuroSCORE in cardiac surgery: how well does it predict operative risk? Heart. 2006;92:1817-20.

19. Hajjar LA, Vincent J-L, Galas FRBG, Nakamura RE, Silva CMP, Santos MH, et al. Transfusion requirements after cardiac surgery: the TRACS randomized controlled trial. JAMA. 2010;304:1559-67. 
APPENDIX TABLE E1. Comparison between matched and unmatched patients

\begin{tabular}{|c|c|c|c|c|}
\hline & $\begin{array}{l}\text { All patients in ANZSCTS database } \\
\text { with ICU admission }(N=27,115)^{*}\end{array}$ & $\begin{array}{l}\text { Unmatched patients } \\
\quad(N=7793)\end{array}$ & $\begin{array}{l}\text { Matched patients } \\
(N=19,304)\end{array}$ & $P$ value \\
\hline Age, y & $68(58-74)$ & $69(60-76)$ & $67(57-75)$ & $<.0001$ \\
\hline Gender $†$ & $1(1-2)$ & $1(1-2)$ & $1(1-2)$ & .07 \\
\hline Ventricular repair $(\%)$ & 38 & 39 & 38 & .3 \\
\hline Coronary artery bypass graft $(\%)$ & 70 & 72 & 69 & $<.0001$ \\
\hline Other cardiac surgery $(\%)$ & 10 & 9 & 11 & $<.0001$ \\
\hline Urgency $\ddagger$ & $1(1-2)$ & $1(1-2)$ & $1(1-2)$ & $<.0001$ \\
\hline New York Heart Association functional class & $2(1-3)$ & $2(1-2)$ & $2(1-3)$ & $<.0001$ \\
\hline Preoperative inotropes & 3 & 3 & 3 & .45 \\
\hline Ejection fraction & $60(50-65)$ & $60(50-65)$ & $60(46-65)$ & $<.0001$ \\
\hline Preoperative dialysis & 1.7 & 1 & 2 & $<.0001$ \\
\hline Body mass index & 28.7 & 28.9 & 28.6 & .06 \\
\hline Allprocscore & $6(2-9)$ & $6(3-9)$ & $5(2-9)$ & $<.0001$ \\
\hline Mortality & 2 & 2 & 2 & .07 \\
\hline
\end{tabular}

Values are given as \%, median (interquartile range), or mean \pm standard deviation. Percentages relate to column population. ANZSCTS, Australia and New Zealand Society of Cardiac and Thoracic Surgeons; $I C U$, intensive care unit. *Matching data was missing for 18 patients. $\dagger 1=$ male, $2=$ female. $\ddagger$ Urgency: $1=$ Elective, $2=$ Urgent, $3=$ Emergency, and $4=$ Salvage. 